\title{
Was ist Nachhaltigkeit?
}

Es ist eigentlich schon lange Zeit für eine Ausgabe der Keramischen Zeitschrift zum Thema Nachhaltigkeit. Schließlich ist die Entwicklung nachhaltiger Prozesse nicht nur eine Notwendigkeit, sondern derzeit auch eine Mammutaufgabe. ,Nachhaltigkeit' ist gewissermaßen ein Zauberwort, denn wenn der Begriff fällt, ist einem die Aufmerksamkeit gesichert. Er wird meist begleitet von Worten wie ,Umweltverträglichkeit', ,Ressourcen und ,Treibhausgasemissionen .

Doch bei der Planung des Heftes fällt auf: Die Definition von ,Nachhaltigkeit' ist viel komplexer. Es ist ein Handlungsprinzip, welches Konzepte, Strategien und unzählige Zielsetzungen in verschiedensten Bereichen unseres Lebens umfasst. Und tatsächlich gibt es die Idee schon seit über 300 Jahren. Ursprünglich auf die Forstwirtschaft bezogen bedeutet Nachhaltigkeit: „Nur so viel Holz schlagen, wie auch nachwachsen kann“. Was für eine Bedeutung hat das also für die Keramikindustrie?

Eine einzelne Ausgabe kann hier unmöglich den Anspruch auf Voll- ständigkeit erheben. Aber sie kann einen Überblick geben und den Sachverhalt aus verschiedenen Perspektiven beleuchten. Wir betrachten deshalb zunächst Strategien, wie die energie- und emissionsintensive Keramikindustrie einen Weg zur Energiewende und Nachhaltigkeit einschlagen kann und was für Hürden sich dabei ergeben. Unter anderem gilt es die Frage zu klären, ob und wie alternativer Energieträger in keramischen Prozessen eingesetzt werden können. Dabei ist es auch interessant, einen Blick auf die Schritte zu werfen, die einige Unternehmen bereits gehen und auf die Lösungen, die ihnen zur Energieeinsparung zur Verfügung stehen.

Auf der anderen Seite liefern keramische Technologien Mittel und Wege, verschiedene Nachhaltigkeitsziele zu erreichen: So sind sie entscheidend bei der Produktion von grünem Wasserstoff, der Funktionalität von Energiespeichern für die E-Mobilität oder der Umwandlung von klimaschädlichem $\mathrm{CO}_{2}$ in einen nutzbaren Rohstoff.

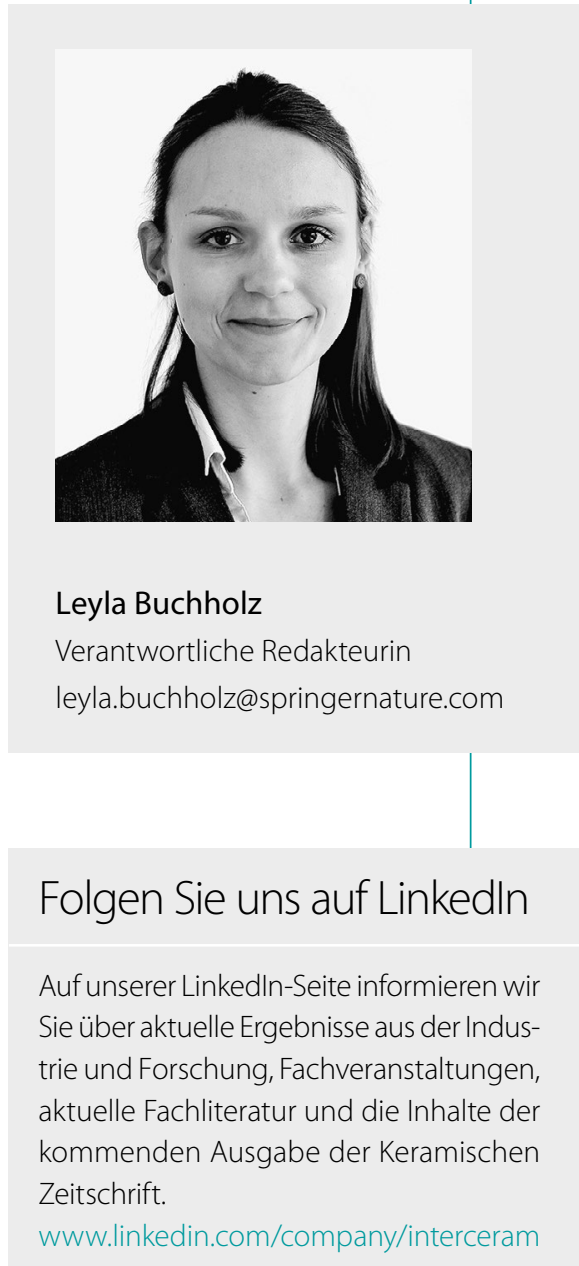

Leyla Buchholz

Verantwortliche Redakteurin

eyla.buchholz@springernature.com

www.linkedin.com/company/interceram 